\title{
Post-synthetic 'click' synthesis of RAFT polymers with pendant self-immolative triazoles
}

\author{
Timothy N. Forder ${ }^{[a]+}$, Peter G. Maschmeyer ${ }^{[a]+}$, Haoxiang Zeng ${ }^{[a]}$ and Dr Derrick A. Roberts ${ }^{*[a, b]}$
}

[a] T. N. Forder, P. G. Maschmeyer, H. Zeng, *Dr D. A. Roberts
Key Centre for Polymers and Colloids, School of Chemistry
The University of Sydney
Sydney NSW 2006, Australia
[b] ${ }^{*}$ Dr D. A. Roberts
Sydney Nano Institute
The University of Sydney
Sydney NSW 2006, Australia
E-mail: derrick.roberts@sydney.edu.au
†Authors contributed equally to this work. Supporting information for this article is given via a link at the end of the document.

\begin{abstract}
Self-immolative linkers offer efficient mechanisms for deprotecting 'caged' functional groups in response to specific stimuli. Herein we describe a convenient 'click' chemistry method for introducing pendant self-immolative linkers to a polymer backbone through post-polymerization modification. The intro duced triazole rings serve both to anchor the stimuli-cleavable trigger groups to the polymer backbone, while also forming a functional part of the selfimmolation cascade. We investigate the polymerization kinetics, postsynthetic modification, and self-immolation mechanism of a model polymer system, and discuss avenues for future studies on polypendant self-immolative triazoles as a modular, stimuli-responsive macromolecule platform.
\end{abstract}

Self-immolative linkers are multicomponent molecules that undergo spontaneous head-to-tail fragmentation through a cascade of cyclization or elimination reactions in a fashion reminiscent of a burning fuse. ${ }^{[1]}$ Such linkers are used to connect a chemical payload to a cleavable protecting group (designated as the 'trigger'), serving to maintain space between the trigger and payload and enhancing the entropic driving force of the deprotection reactions. ${ }^{[2]}$ As such, self-immolative linkers are useful motifs for controlling the unmasking of reactive functional groups in response to external stimuli. This behavior has formed the basis of prodrug ${ }^{[3]}$ and other controlled-release platforms, ${ }^{[4]}$ chemical sensors ${ }^{[5]}$ and amplifiers, ${ }^{[6]}$ and rapidly degradable bulk materials. ${ }^{[7]}$

The unique qualities of self-immolative linkers have drawn significant attention from the polymer chemistry community over the past decade, most notably in the development self-immolative polymers (SIPs ${ }^{[8]}$ and related macromolecules (e.g., dendrimers and brushes). ${ }^{\left[{ }^{[9]}\right.}$ SIPs are low ceiling temperature polymers that can be formed by connecting together several self-immolative linkers to form a continuous chain that will undergo spontaneous depolymerization upon cleavage of the trigger end-group. In addition to forming the backbones of polymer architectures, selfimmolative motifs have recently been used as stimuli-responsive pendant groups in synthetic macromolecules. For instance, Liu and co-workers have used amphiphilic polymers with selfimmolative pendants to construct a range of stimuli-responsive polymersome nanocarriers. ${ }^{[10]}$ In these systems, the selfimmolative pendant groups control the permeability of the polymersomes through post-assembly cross-linking reactions, ${ }^{[11]}$ thereby gating the movement of small molecules across their membranes. Synthesis of the polymers is straightforward via RAFT polymerization, enabling the formation of block copolymer architectures. However, the self-immolative pendant groups are typically introduced to the polymers via isocyanate or blocked isocyanate precursors, requiring anhydrous conditions and oftentimes high temperatures (e.g., $80-110^{\circ} \mathrm{C}$ for thermal deblocking of a phenolic isocyanate). ${ }^{[12]}$ Consequently, there is scope for developing milder ways to introduce self-immolative linkers into polymeric architectures that avoid isocyanate or isocyanate-equivalent precursors at the critical post-synthetic modification step.

Recently, our group developed a methodology for preparing self-immolative triazole (SIT) linkers using the copper(I) catalyzed azide-alkyne cycloaddition (CuAAC) reaction, ${ }^{[13]}$ inspired by earlier work from the groups of Hayes and Russell[14] and Bertrand. ${ }^{[15]}$ Our linker design features a diamine-derived cyclisation spacer connected in series to a 1,4-triazole bridge, which undergoes rapid elimination under basic conditions (Scheme 1). Conveniently, the SIT linker is formed by the mild and efficient CuAAC reaction between a propargylic carbamate and an azide-bearing trigger precursor (e.g., azide A, Scheme 2). Considering the popularity of the CUAAC reaction for the postsynthetic functionalization of polymers, ${ }^{[16]}$ we anticipate that our methodology could be readily adapted for the post-synthetic introduction of self-immolative pendant groups onto polymers to generate stimuli-responsive macromolecules.

To demonstrate proof-of-concept, we prepared $\mathbf{P} \mathbf{1}$, a random copolymer of ethyl acrylate and an alkyne-functionalized monomer (M1), and studied its post-synthetic modification using azide $\mathbf{A}$ under CuAAC conditions to form polymer P2 (Scheme 1). Monomer M1 was designed according to previous observations that dimethyl substitution at the propargylic position generates a triazole product that, once triggered, undergoes rapid 1,4elimination at a rate comparable to the diamine cyclization step. ${ }^{\text {[13- }}$ 14] Maximizing the rate of triazole 1,4-elimination permits determination of the upper rate-limit of self-immolative release in this system, which can provide important kinetics information for the design of future controlled release systems. ${ }^{[17]}$ Additionally, allyl-protected trigger-azide $\mathbf{A}$ was selected as a model protecting group due to its well characterized self-immolation behavior in previous studies ${ }^{[13]}$ and its extremely rapid deprotection under mild conditions, which facilitates in situ kinetics studies by ${ }^{1} \mathrm{H}$ NMR spectroscopy. 


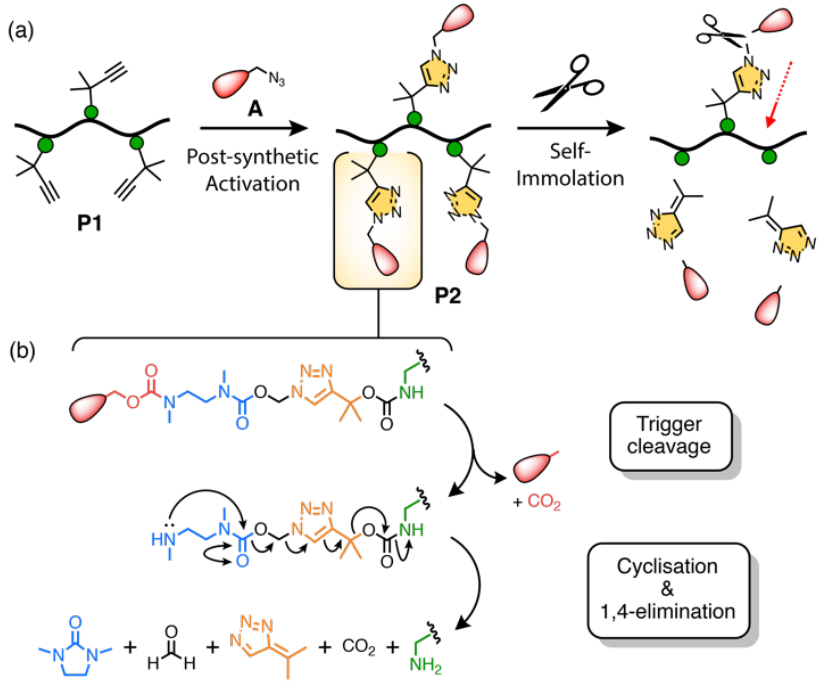

Scheme 1. (a) General scheme illustrating post-synthetic modification and selfimmolative uncaging of poly-pendant SITs. (b) Self-immolation mechanism of the SIT linkers. Trigger cleavage exposes an unstable secondary amine linker that undergoes a spontaneous cyclisation and 1,4-elimination sequence to uncage the primary amine residue. Proton transfers are not shown explicitly.

Monomer M1 was prepared in three steps from 2-methyl-3butyn-2-ol (1) (Scheme 2), starting with the formation of active carbonyl ester 2 using 1,1-carbonyldiimidazole followed by treatment with 2-ethanolamine to afford carbamate 3 in good yield (78\% yield over 2 steps). $\mathrm{N}$-carbamoylation was observed exclusively under these conditions, with no unwanted carbonate formation at the alcohol site. Subsequent esterification of $\mathbf{3}$ with acryloyl chloride at -20 to $0{ }^{\circ} \mathrm{C}$ furnished monomer M1 in poor yield $(\sim 37 \%)$. We were perplexed by the consistently low yield of the esterification reaction with acryloyl chloride, which was initially ascribed to degradation of the monomer via olefin hydrochlorination. ${ }^{[18]}$ However, TLC analysis did not indicate significant formation of side products (SI, Figure S10), and we did not identify plausible side-products by MS during the reaction. Nonetheless, purification of the monomer by silica gel chromatography afforded a highly pure monomer that was characterized in full by NMR and HRMS (SI, Section S3).

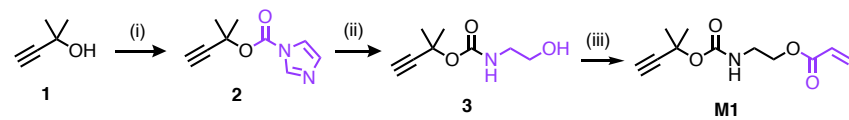

$$
\begin{aligned}
& \underset{\text { (iv) }}{\stackrel{\text { CPDTC }}{\mathrm{C}_{12} \mathrm{H}_{25} \mathrm{~S}{ }_{\mathrm{S}}^{\mathrm{S}} \mathrm{X}_{\mathrm{CN}}} \mathrm{C}_{12} \mathrm{H}_{25} \mathrm{~S}}
\end{aligned}
$$

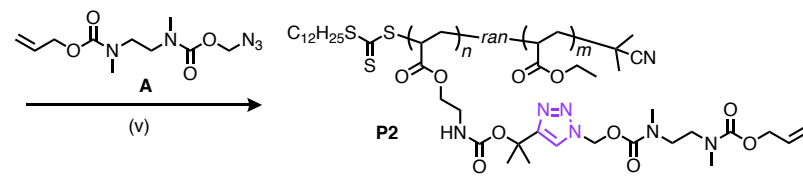

Scheme 2. Overview of monomer synthesis, polymerization and post-synthetic modification steps. Reagents and conditions: (i) $\mathrm{CDI}, \mathrm{CH}_{2} \mathrm{Cl}_{2}$, r.t. $43 \mathrm{~h},>99 \%$. (ii) 2-ethanolamine, $\mathrm{CH}_{2} \mathrm{Cl}_{2}, 0{ }^{\circ} \mathrm{C}$ to r.t., $20 \mathrm{~h}, 78 \%$. (iii) Acryloyl chloride, $\mathrm{Et}_{3} \mathrm{~N}$, 20 to $0{ }^{\circ} \mathrm{C}$ to r.t., $3.5 \mathrm{~h}, 37 \%$. (iv) Ethyl acrylate, ZnTPP, 1,4-dioxane, r.t., $90 \mathrm{~min}$ (55\% conversion). (v) $\mathrm{CuSO}_{4} \cdot 5 \mathrm{H}_{2} \mathrm{O} /$ sodium ascorbate, DMF, $50{ }^{\circ} \mathrm{C}, 24 \mathrm{~h}$.
Polymer P1 was prepared by PET-RAFT polymerization ${ }^{[19]}$ of M1 with ethyl acrylate (1:9 feed ratio targeting $\sim$ DP100) in DMSO using CPDTC as the chain transfer agent and zinc tetraphenylporphyrin (ZnTPP) as the photocatalyst (Scheme 2). A $1: 9$ feed ratio was chosen to limit potential alkyne photocrosslinking during polymerization, and to reduce the possibility of ester to amide crosslinking reactions along the polymer backbone following self-immolation, ${ }^{[11]}$ which would complicate kinetics experiments. To confirm the controlled radical polymerization of M1 with ethyl acrylate, we followed the polymerization kinetics by ${ }^{1} \mathrm{H}$ NMR and SEC (SI, Section S4). After an induction period of approximately $30 \mathrm{~min}$, the polymerization exhibited features of a well-controlled RAFT mechanism, including a linear increase in $M_{\mathrm{n}}$ with monomer conversion, narrow dispersities $(\theta \leq 1.2)$, and a linear semi-logarithmic rate plot consistent with pseudo first-order kinetics (Figure 1 and Figure S13). However, above $70 \%$ conversion the kinetics deviated from first-order behavior and the SEC traces became progressively broader with the appearance of a high molecular weight shoulder (Figure $1 \mathrm{~b}$ ). We attribute this to incipient photoinduced cross-linking of the alkyne groups as the free monomer is depleted. Based on these data, we concluded that limiting the polymerization conversion to $<70 \%$ at this feed ratio is necessary to minimize cross-linking and thus produce polymers with monomodal and uniform molecular weight distributions.
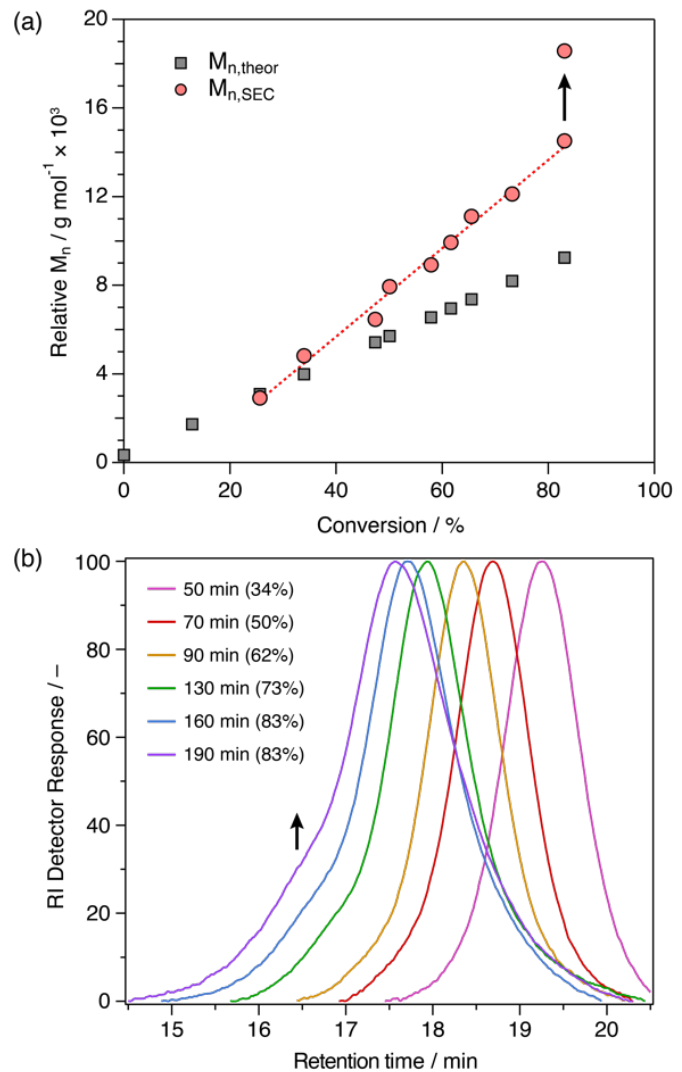

Figure 1. Data for the PET-RAFT polymerization of monomer 4 to form polymer 5. (a) Molecular weight versus conversion plot showing linear evolution of $M_{\mathrm{n}, \mathrm{SEC}}$ (DMAc/LiBr) with increasing conversion, up to $\sim 83 \%$ conversion. $M_{n, \text { theor }}$ is calculated from NMR-derived conversion values. Black arrow indicates sudden increase in $M_{n, S E C}$ due to putative crosslinking. (b) Representative SEC traces (conversions shown in parentheses) showing narrow and monomodal molecular weight distributions up to $\sim 70 \%$ conversion, after which time a high molecular weight shoulder, attributed to incipient crosslinking, appears (black arrow). 
To study post-polymerization modification, a sample of $\mathbf{P} \mathbf{1}$ was prepared at $55 \%$ conversion $\left(M_{\mathrm{n}, \mathrm{NMR}}=7,000 \mathrm{~g} \mathrm{~mol}^{-1}, D=1.13\right.$, $n=5, m=55$; see SI, Section S4). ${ }^{1} \mathrm{H}$ NMR integration revealed a repeat unit stoichiometry of $n: \underline{m}=1: 11$ - lower than the 1:9 monomer feed ratio, suggesting $\overline{\mathbf{M}} \mathbf{1}$ is slightly less reactive than ethyl acrylate toward propagating radicals. Once purified, SIT pendants were attached to $\mathbf{P} \mathbf{1}$ by treating the polymer with azide A ( 2-3 equiv. per alkyne) using a copper(II) sulfate-sodium ascorbate catalyst system in DMF at $50{ }^{\circ} \mathrm{C}$ for $24 \mathrm{~h}$ under air. The hydrophobicity of the polymer permitted aqueous work-up by dissolution in EtOAc and washing with aqueous EDTA ( $2 \% \mathrm{w} / \mathrm{v})$, followed by precipitation into diethyl ether to afford purified $\mathbf{P 2}$. Successful post-synthetic modification of P1 was confirmed by ${ }^{1} \mathrm{H}$ NMR, which revealed peaks characteristic of the SIT pendants (Figure 2b). Integration revealed an estimated repeat unit stoichiometry of $n: \underline{m}=1: 13$, which corresponds to a $~ 85 \%$ grafting efficiency under these conditions. Additionally, DOSY NMR confirmed attachment of the SIT linkers to the backbone, evidenced by co-diffusion of both sets of characteristic peaks (Figure 2c), and HSQC analysis verified key ${ }^{1} \mathrm{H}$ and ${ }^{13} \mathrm{C}$ assignments (SI, Section S4.2). The SEC trace of P2 (SI, Figure S23) showed a very slight shift towards shorter retention time and an increase in dispersity $(\Theta=1.17)$ compared to $\mathbf{P} \mathbf{1}$, consistent with a small increase in size due to the SIT pendants and possible further crosslinking of the alkyne groups (e.g., via adventitious $\mathrm{Cu}(\mathrm{I})$-catalyzed Glaser coupling in the presence of $\left.\mathrm{O}_{2}\right){ }^{[20]}$ Future work will seek to optimize our CuAAC coupling methodology using anoxic conditions and rapid dielectric heating. ${ }^{[21]}$
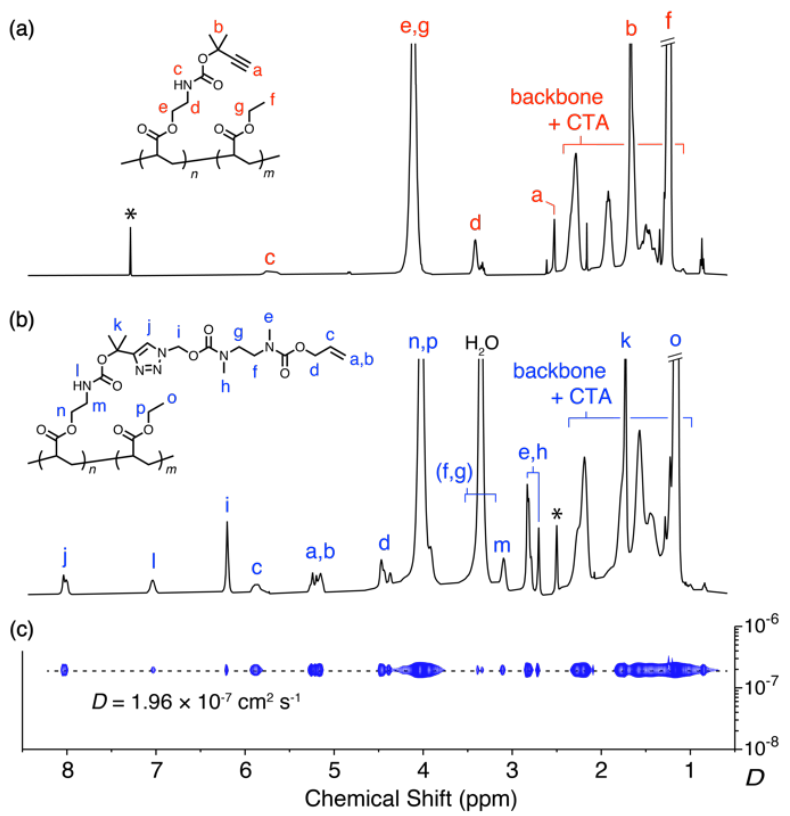

Figure 2. ${ }^{1} \mathrm{H}$ NMR spectra $\left(400 \mathrm{MHz}, 298 \mathrm{~K}\right.$ ) of (a) $\mathbf{P} 1$ in $\mathrm{CDCl}_{3}$, (b) $\mathbf{P} 2$ in DMSO- $d_{6}\left(\mathrm{CDCl}_{3}\right.$ spectrum in SI, Figure S18) and (c) corresponding ${ }^{1} \mathrm{H}$ DOSY spectrum (units of $D$ are $\mathrm{cm}^{2} \mathrm{~s}^{-1}$; expanded spectrum in SI, Figure S21).

Self-immolation kinetics of polymer $\mathbf{P 2}$ were measured by ${ }^{1} \mathrm{H}$ NMR spectroscopy in DMSO- $d_{6} / \mathrm{D}_{2} \mathrm{O} 95: 5$ at $60{ }^{\circ} \mathrm{C}(\mathrm{SI}$, Section S5). Trigger cleavage was initiated by the addition of catalytic $\mathrm{Pd}\left(\mathrm{PPh}_{3}\right)_{4}$ (5-10 mol\%) in the presence of morpholine (1012 equiv. per SIT pendant) to regenerate the catalyst and render all base-mediated reaction steps pseudo first-order. As noted previously, the linker was stable under basic conditions in the absence of palladium, and only underwent chemospecific degradation when exposed to the catalyst. All three stages of the self-immolation cascade were distinguishable by NMR, allowing estimation of pseudo-first-order rate constants $\left(k_{\text {obs }}\right)$ for each step (Scheme 3; SI, Section S5.2). Rate constants for trigger removal and cyclisation were on the order of $10^{-2} \mathrm{~s}^{-1}$ and $10^{-3} \mathrm{~s}^{-1}$ respectively, agreeing closely with our previous measurements using small molecule SIT models. ${ }^{[13]}$ Cyclization of the diamine spacer and 1,4-elimination of the triazole spacer were observed to occur at the same rate. This is consistent with the high rate of 1,4-elimination for dimethyl substitution at the triazole $\alpha$-methine position, which is understood to stabilize the build-up of positive charge at this carbon during 1,4-elimination. ${ }^{[14]}$ These kinetics also confirm that cyclization is the rate-determining step of the cascade, and that faster self-immolation can only be achieved by employing more rapidly cyclizing spacers. ${ }^{[22]}$ However, it should be quite straightforward to access slower self-immolation rates by employing more electron deficient substituents at the triazole $\alpha$ methine position, which could enable rate tuning for controlled release in the hours-to-days kinetics regime.
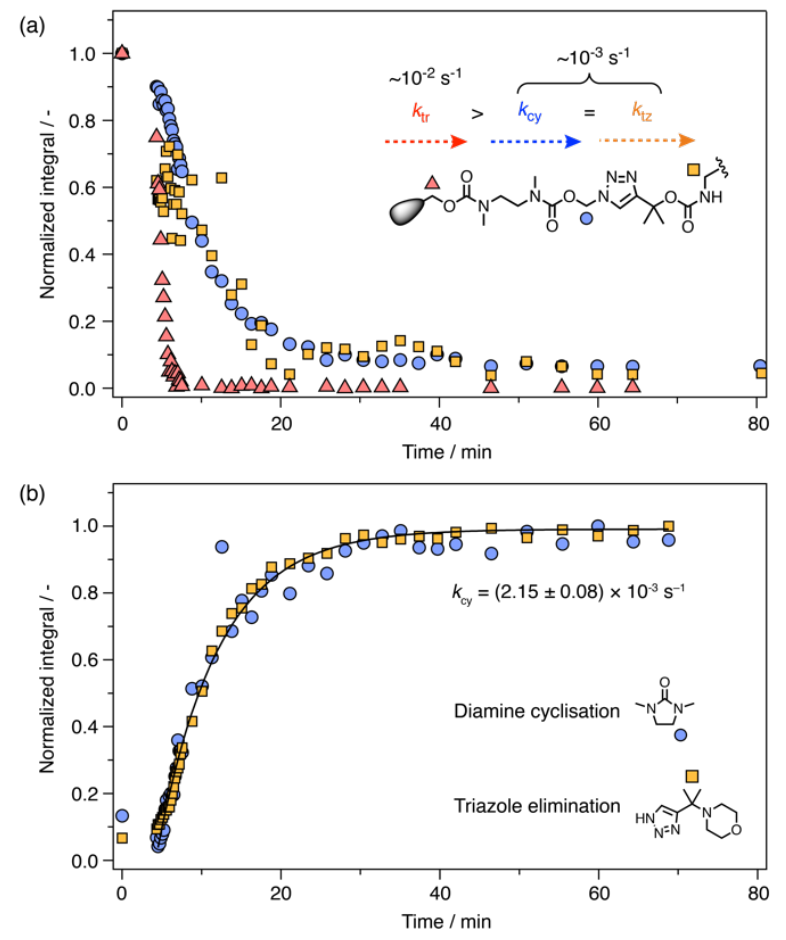

Figure 3. Self-immolation kinetics of polymer $\mathbf{P} 2$ measured by in situ ${ }^{1} \mathrm{H}$ NMR spectroscopy (DMSO- $d_{6} / \mathrm{D}_{2} \mathrm{O}=95: 5,400 \mathrm{MHz}, 333 \mathrm{~K}$ ). Markers on chemical structures indicate proton signals that were tracked for kinetics analysis. (a) Degradation of the linker components. (b) Appearance of the cyclization and 1,4 -elimination products. The black line is fitted to the yellow squares according to a first-order (monoexponential) kinetics model.

In conclusion, we have demonstrated a convenient postsynthetic strategy for attaching triazole-based self-immolative linkers onto a polymeric backbone using the CuAAC reaction. To the best of our knowledge this is the first example of a selfimmolative triazole featuring within a polymeric architecture, and therefore represents a new way to introduce stimuli-responsivity to synthetic polymers through covalent post-modification. This strategy is inherently modular due to the possibility of substitutional variations within the linker design (e.g., different trigger groups and substitution of the triazole $\alpha$-methine position) and the versatility of RAFT polymerization both in terms of monomer scope and polymer architecture. In future work we will 
examine the range of monomers that can be caged and released using this chemistry, and the possibility of tuning the rate of selfimmolative release through substituent effects within the linker. There is also broad scope for preparing block copolymer architectures featuring pendant SITs as building blocks for selfassembly into stimuli-responsive polymer nanostructures with tunable controlled release properties.

\section{Acknowledgements}

The authors acknowledge financial support from the Australian Research Council Discovery Early-Career Researcher Award scheme (DE190100797), The School of Chemistry, and the DVCR Office at The University of Sydney. The authors also acknowledge the facilities and the scientific and technical assistance of Sydney Analytical, a core research facility at the University of Sydney. We thank Dr lan Luck (NMR) and Dr Nicholas Proschogo (mass spectrometry) for technical assistance, and $\mathrm{Mr}$ Karen Hakobyan and $\mathrm{Dr}$ Thomas Gegenhuber for maintenance of the analytical SEC systems.

Keywords: self-immolative linkers $•$ reaction kinetics $\cdot$ click chemistry $\cdot$ RAFT polymerization $\cdot$ post-synthetic modification

[1] a) A. Alouane, R. Labruere, T. Le Saux, F. Schmidt, L. Jullien, Angew. Chem. Int. Ed. 2015, 54, 7492-7509; b) J. Yan, S. Lee, A. Zhang, J. Yoon, Chem. Soc. Rev. 2018, 47, 6900-6916; c) M. Gisbert-Garzarán, M. Manzano, M. Vallet-Regí, Chem. Eng. J. 2018, 340, 24-31.

[2] C. A. Blencowe, A. T. Russell, F. Greco, W. Hayes, D. W. Thornthwaite, Polym. Chem. 2011, 2, 773-790.

[3] a) P. L. Carl, P. K. Chakravarty, J. A. Katzenellenbogen, J. Med. Chem. 1981, 24, 479-480; b) F. M. de Groot, W. J. Loos, R. Koekkoek, L. W. van Berkom, G. F. Busscher, A. E. Seelen, C. Albrecht, P. de Bruijn, H. W. Scheeren, J. Org. Chem. 2001, 66, 8815-8830; c) C. Zang, H. Wang, T. Li, Y. Zhang, J. Li, M. Shang, J. Du, Z. Xi, C. Zhou, Chem. Sci. 2019, 10, 8973-8980; d) B. Wang, S. Van Herck, Y. Chen, X. Bai, Z. Zhong, K. Deswarte, B. N. Lambrecht, N. N. Sanders, S. Lienenklaus, H. W. Scheeren, S. A. David, F. Kiessling, T. Lammers, B. G. De Geest, Y. Shi, J. Am. Chem. Soc. 2020, 142, 12133-12139.

[4] a) K. M. Schmid, L. Jensen, S. T. Phillips, J. Org. Chem. 2012, 77, 43634374; b) F. Seidi, R. Jenjob, D. Crespy, Chem. Rev. 2018, 118, 39654036; c) X. Ji, Z. Pan, B. Yu, L. K. De La Cruz, Y. Zheng, B. Ke, B. Wang, Chem. Soc. Rev. 2019, 48, 1077-1094; d) S. M. Heuchan, B. Fan, J. J. Kowalski, E. R. Gillies, H. A. L. Henry, J. Agric. Food Chem 2019, 67, 12720-12729; e) Y. Duan, Y. Wang, X. Li, G. Zhang, G. Zhang, J. Hu, Chem. Sci. 2020, 11, 186-194; f) T. D. Harrison, O. Yunyaeva, A. Borecki, C. C. Hopkins, J. R. de Bruyn, P. J. Ragogna, E. R. Gillies, Biomacromolecules 2020, 21, 152-162.

[5] a) J. Hu, S. Liu, Macromolecules 2010, 43, 8315-8330; b) R. Labruère, A. Alouane, T. Le Saux, I. Aujard, P. Pelupessy, A. Gautier, S. Dubruille, F. Schmidt, L. Jullien, Angew. Chem. Int. Ed. 2012, 51, 9344-9347; c) H.Y. Huang, S.-Y. Fan, E.-H. Chang, C. H. Lam, Y.-C. Lin, X.-H. Lin, N. K. Gupta, K.-T. Tan, Anal. Chem. 2020, 92, 15463-15471; d) Q. Li, Y. Wu, Y. Liu, L. Shangguan, B. Shi, H. Zhu, Organic Letters 2020, 22, 66626666 .

[6] a) M. Avital-Shmilovici, D. Shabat, Soft Matter 2010, 6, 1073-1080; b) R. Perry-Feigenbaum, E. Sella, D. Shabat, Chem. Eur. J. 2011, 17, 1212312128; c) N. Karton-Lifshin, D. Shabat, New. J. Chem. 2012, 36, 386393; d) M. E. Roth, O. Green, S. Gnaim, D. Shabat, Chem. Rev. 2016, 116, 1309-1352

[7] a) W. Seo, S. T. Phillips, J. Am. Chem. Soc. 2010, 132, 9234-9235; b) A. M. DiLauro, G. G. Lewis, S. T. Phillips, Angew. Chem. Int. Ed. 2015, 54, 6200-6205; c) K. Yeung, H. Kim, H. Mohapatra, S. T. Phillips, J. Am. Chem. Soc. 2015, 137, 5324-5327; d) A. Rahimi, J. M. García, Nat. Rev. Chem. 2017, 1, 0046.
[8] a) A. Sagi, R. Weinstain, N. Karton, D. Shabat, J. Am. Chem. Soc. 2008, 130, 5434-5435; b) G. I. Peterson, M. B. Larsen, A. J. Boydston, Macromolecules 2012, 45, 7317-7328; c) B. Fan, J. F. Trant, A. D. Wong, E. R. Gillies, J. Am. Chem. Soc. 2014, 136, 10116-10123; d) J. A. Kaitz, O. P. Lee, J. S. Moore, MRS Commun. 2015, 5, 191-204; e) Y. Xiao, X. Tan, Z. Li, K. Zhang, J. Mater. Chem. B 2020, 8, 6697-6709; f) Q. E. A. Sirianni, E. R. Gillies, Polymer 2020, 202, 122638.

[9] a) S. Gnaim, D. Shabat, Acc. Chem. Res. 2014, 47, 2970-2984; b) G. Liu, G. Zhang, J. Hu, X. Wang, M. Zhu, S. Liu, J. Am. Chem. Soc. 2015, 137, 11645-11655; c) X. Tan, B. B. Li, X. Lu, F. Jia, C. Santori, P. Menon, H. Li, B. Zhang, J. J. Zhao, K. Zhang, J. Am. Chem. Soc. 2015, 137, 6112-6115; d) Y. Xiao, H. Li, B. Zhang, Z. Cheng, Y. Li, X. Tan, K. Zhang, Macromolecules 2018, 51, 2899-2905; e) Y. Xiao, Y. Li, B. Zhang, H. Li, Z. Cheng, J. Shi, J. Xiong, Y. Bai, K. Zhang, ACS Macro Lett. 2019, 8, 399-402.

[10] a) X. Wang, G. Liu, J. Hu, G. Zhang, S. Liu, Angew. Chem. Int. Ed. 2014, 53, 3138-3142; b) Z. Deng, Y. Qian, Y. Yu, G. Liu, J. Hu, G. Zhang, S. Liu, J. Am. Chem. Soc. 2016, 138, 10452-10466; c) J. Tan, Z. Deng, G. Liu, J. Hu, S. Liu, Biomaterials 2018, 178, 608-619; d) X. Wang, C. Yao, G. Zhang, S. Liu, Nat. Comm. 2020, 11, 1524

[11] a) D. A. Roberts, B. S. Pilgrim, J. R. Nitschke, Chem. Soc. Rev. 2018, 47, 626-644; b) H. Zeng, L. Stewart-Yates, L. M. Casey, N. Bampos, D. A. Roberts, ChemPlusChem 2020, 85, 1249-1269.

[12] M. S. Rolph, A. L. J. Markowska, C. N. Warriner, R. K. O'Reilly, Polym. Chem. 2016, 7, 7351-7364.

[13] D. A. Roberts, B. S. Pilgrim, T. N. Dell, M. M. Stevens, Chem. Sci. 2020, $11,3713-3718$.

[14] C. A. Blencowe, D. W. Thornthwaite, W. Hayes, A. T. Russell, Org. Biomol. Chem. 2015, 13, 8703-8707.

[15] a) M. Mondon, R. Delatouche, C. Bachmann, G. Frapper, C. Len, P. Bertrand, Eur. J. Org. Chem. 2011, 2011, 2111-2119; b) R. Delatouche, M. Mondon, A. Gil, G. Frapper, C. Bachmann, P. Bertrand, Tetrahedron 2011, 67, 401-407.

[16] a) R. A. Evans, Aust. J. Chem. 2007, 60, 384-395; b) W. H. Binder, R. Sachsenhofer, Macromol. Rapid Commun. 2008, 29, 952-981; c) P. Leophairatana, C. C. De Silva, J. T. Koberstein, J. Polym. Sci. A. 2018, 56, 75-84.

[17] A. Alouane, R. Labruère, T. Le Saux, I. Aujard, S. Dubruille, F. Schmidt, L. Jullien, Chem. Eur. J. 2013, 19, 11717-11724.

[18] M. Movsisyan, T. S. A. Heugebaert, B. I. Roman, R. Dams, R. Van Campenhout, M. Conradi, C. V. Stevens, Chem. Eur. J. 2018, 24, 1177911784.

[19] a) S. Shanmugam, J. Xu, C. Boyer, Macromolecules 2014, 47, 49304942; b) J. Phommalysack-Lovan, Y. Chu, C. Boyer, J. Xu, Chem. Commun. 2018, 54, 6591-6606.

[20] P. Leophairatana, S. Samanta, C. C. De Silva, J. T. Koberstein, J. Am. Chem. Soc. 2017, 139, 3756-3766.

[21] a) D. A. Roberts, T. W. Schmidt, M. J. Crossley, S. Perrier, Chem. Eur. J. 2013, 19, 12759-12770; b) D. A. Roberts, M. J. Crossley, S. Perrier, Polym. Chem. 2014, 5, 4016-4021.

[22] a) E. K. Y. Chen, R. A. McBride, E. R. Gillies, Macromolecules 2012, 45, 7364-7374; b) S. Huvelle, A. Alouane, T. Le Saux, L. Jullien, F. Schmidt, Org. Biomol. Chem. 2017, 15, 3435-3443; c) A. Dal Corso, V. Borlandelli, C. Corno, P. Perego, L. Belvisi, L. Pignataro, C. Gennari, Angew. Chem. Int. Ed. 2020, 59, 4176-4181. 